\title{
Teaching NeuroImages: Pseudopathologic brain parenchymal enhancement due to vascular compression in parotid tumor
}

Bernardo Corrêa de Almeida Teixeira, MD, PhD, Marianna Cioni, MD, Debora Brighente Bertholdo, MD, Luiz Otávio de Mattos Coelho, MD, and Dante Luiz Escuissato, MD, PhD

Neurology ${ }^{\circledR}$ 2020;94:e1778-1779. doi:10.1212/WNL.0000000000009300

Figure 1 CT angiography

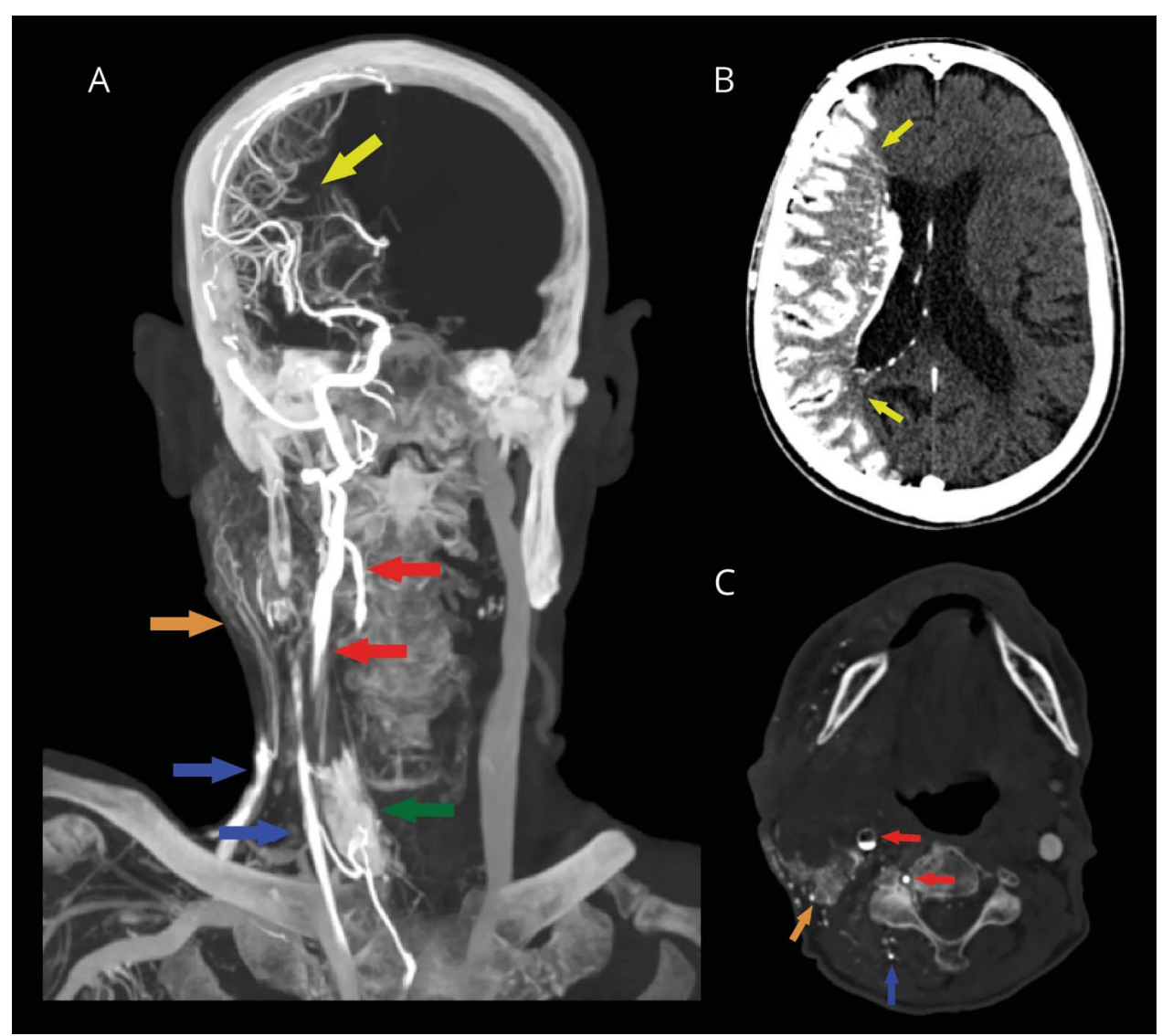

Coronal (A) and axial (B, C) CT angiography done by right side antecubital vein injection reveals jugular and cervical veins reflux (blue arrows) reaching the parotid tumor (orange arrow), with opacification of carotid and vertebral arteries (red arrows) and enhancement of intracranial compartment (yellow arrow). Also note hemithyroid contrast reflux (green arrow).

A 74-year-old man with a history of invasive right parotid gland adenocarcinoma presented with acute onset left hemiparesis. CT angiography showed abnormal cervical and intracranial vascular reflux with enhancement of right middle cerebral artery territory (figure 1). Vascular anomalies were suspected but digital subtraction angiography was normal. There was spontaneous resolution of the symptoms and a follow-up CT showed normalization of the imaging findings (figure 2). Pseudopathological brain parenchymal enhancement has been described rarely, usually as an

\section{Correspondence}

Dr. Teixeira

berteixeira@gmail.com

\section{MORE ONLINE}

\section{$\rightarrow$ Teaching Slides}

links.lww.com/WNL/

B75

From the Hospital de Clínicas (B.C.d.A.T., M.C., D.L.E.), Federal University of Paraná; and Clínica Diagnóstico Avançado Por Imagem—DAPI (D.B.B., L.O.d.M.C.), Curitiba, Paraná, Brazil. Go to Neurology.org/N for full disclosures. Funding information and disclosures deemed relevant by the authors, if any, are provided at the end of the article. 
Figure 2 Follow-up CT

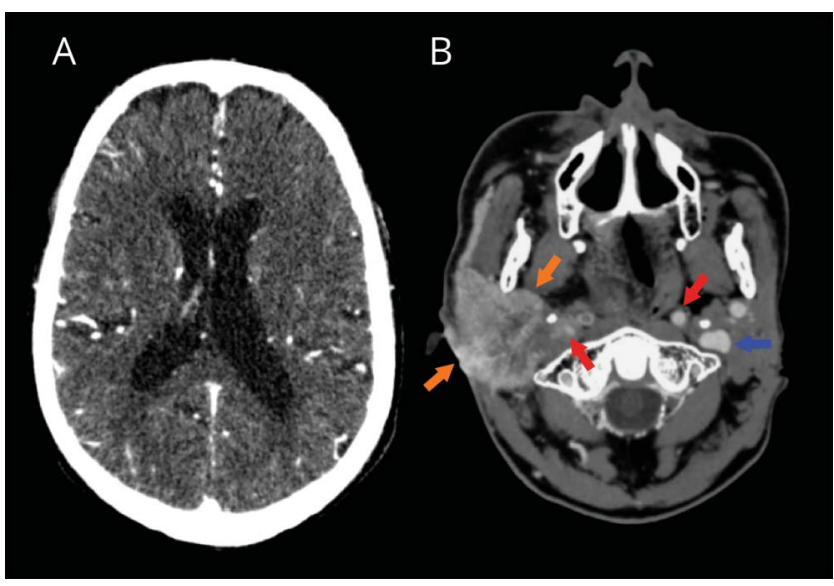

Axial CT with contrast done by left side vein injection shows normal brain opacification (A) and right parotid tumor (orange arrows) involving the right internal carotid artery (red arrows) and no discernible right jugular vein (B). Note normal opacification of left side carotid artery (red arrows) and jugular vein (blue arrow)

incidental finding in asymptomatic patients, and mainly related to venous compression, development of collateral vessels, and incompetent venous valves. ${ }^{1,2}$

\section{Study funding}

No targeted funding reported.

\section{Disclosure}

B.C.A. Teixeira, M. Cioni, D. Bertholdo, L.O.M. Coelho, and D.L. Escuissato report no relevant disclosures. Go to Neurology.org/N for full disclosures.
Appendix Authors

\begin{tabular}{|c|c|c|}
\hline Name & Location & Contribution \\
\hline $\begin{array}{l}\text { Bernardo } \\
\text { Corrêa de } \\
\text { Almeida } \\
\text { Teixeira, MD, } \\
\text { PhD }\end{array}$ & $\begin{array}{l}\text { Hospital de Clínicas, } \\
\text { Paraná, Brazil }\end{array}$ & $\begin{array}{l}\text { Study concept and } \\
\text { design, image analysis } \\
\text { and interpretation, } \\
\text { drafting of the } \\
\text { manuscript }\end{array}$ \\
\hline $\begin{array}{l}\text { Marianna Cioni, } \\
\text { MD }\end{array}$ & $\begin{array}{l}\text { Hospital de Clínicas, } \\
\text { Paraná, Brazil }\end{array}$ & $\begin{array}{l}\text { Study concept and } \\
\text { design, image analysis } \\
\text { and interpretation, and } \\
\text { drafting of the } \\
\text { manuscript }\end{array}$ \\
\hline $\begin{array}{l}\text { Debora } \\
\text { Brighente } \\
\text { Bertholdo, MD }\end{array}$ & $\begin{array}{l}\text { Clínica Diagnóstico } \\
\text { Avançado Por } \\
\text { Imagem-DAPI, Curitiba, } \\
\text { Paraná, Brazil }\end{array}$ & $\begin{array}{l}\text { Image analysis and } \\
\text { interpretation }\end{array}$ \\
\hline $\begin{array}{l}\text { Luiz Otávio de } \\
\text { Mattos Coelho, } \\
\text { MD }\end{array}$ & $\begin{array}{l}\text { Clínica Diagnóstico } \\
\text { Avançado Por } \\
\text { Imagem-DAPI, Curitiba, } \\
\text { Paraná, Brazil }\end{array}$ & $\begin{array}{l}\text { Image analysis and } \\
\text { interpretation }\end{array}$ \\
\hline $\begin{array}{l}\text { Dante Luiz } \\
\text { Escuissato, MD, } \\
\text { PhD }\end{array}$ & $\begin{array}{l}\text { Hospital de Clínicas, } \\
\text { Paraná, Brazil }\end{array}$ & $\begin{array}{l}\text { Study concept and } \\
\text { design, image analysis } \\
\text { and interpretation, } \\
\text { drafting of the } \\
\text { manuscript, and final } \\
\text { approval }\end{array}$ \\
\hline
\end{tabular}

\section{References}

1. Chen JY, Mamourian AC, Messe SR, Wolf RL. Pseudopathological brain parenchymal enhancement due to venous reflux from left-sided injection and brachiocephalic vein narrowing. AJNR Am J Neuroradiol 2010;31:86-87.

2. Kim YK, Sung YM, Hwang KH, Cho EK, Choi HY. Pseudopathologic vertebral body enhancement in the presence of superior vena cava obstruction on computed tomography. Spine J 2015;15:1295-1301. 


\section{Neurology}

\section{Teaching NeuroImages: Pseudopathologic brain parenchymal enhancement due to}

vascular compression in parotid tumor

Bernardo Corrêa de Almeida Teixeira, Marianna Cioni, Debora Brighente Bertholdo, et al. Neurology 2020;94;e1778-e1779 Published Online before print March 27, 2020

DOI 10.1212/WNL.0000000000009300

This information is current as of March 27, 2020

Updated Information \& Services

References

Citations

Subspecialty Collections

Permissions \& Licensing

Reprints including high resolution figures, can be found at: http://n.neurology.org/content/94/16/e1778.full

This article cites 2 articles, 1 of which you can access for free at: http://n.neurology.org/content/94/16/e1778.full\#ref-list-1

This article has been cited by 2 HighWire-hosted articles: http://n.neurology.org/content/94/16/e1778.full\#\#otherarticles

This article, along with others on similar topics, appears in the following collection(s):

All Cerebrovascular disease/Stroke

http://n.neurology.org/cgi/collection/all_cerebrovascular_disease_strok e

All Imaging

http://n.neurology.org/cgi/collection/all_imaging

All Oncology

http://n.neurology.org/cgi/collection/all_oncology

CT

http://n.neurology.org/cgi/collection/ct

Other cerebrovascular disease/ Stroke

http://n.neurology.org/cgi/collection/other_cerebrovascular_disease_s troke

Information about reproducing this article in parts (figures,tables) or in its entirety can be found online at:

http://www.neurology.org/about/about_the_journal\#permissions

Information about ordering reprints can be found online:

http://n.neurology.org/subscribers/advertise

Neurology ${ }^{\circledR}$ is the official journal of the American Academy of Neurology. Published continuously since 1951, it is now a weekly with 48 issues per year. Copyright (C 2020 American Academy of Neurology. All rights reserved. Print ISSN: 0028-3878. Online ISSN: 1526-632X.

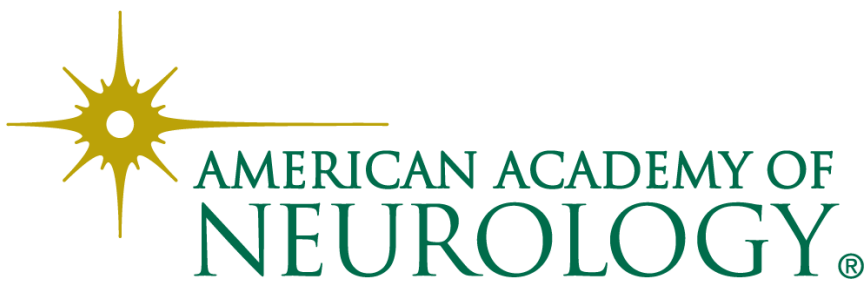

